\title{
论 文纪念《中国科学》创刊70周年专辑
}

\section{面向数据驱动材料设计的专用数据库及信息管理 系统}

宋晓艳", 刘东, 刘雪梅, 郭凯, 毛斐

北京工业大学材料科学与工程学院, 新型功能材料教育部重点实验室, 北京 100124

*E-mail: xysong@bjut.edu.cn

收稿日期: 2019-10-30; 接受日期: 2019-12-11; 网络版发表日期: 2020-03-30

国家重点研发计划(编号: 2018YFB0703902, 2016YFB0700503)和国家杰出青年科学基金(编号: 51425101)资助项目

摘要随着材料设计逐渐向“数据驱动”的方向发展, 数据集的规模和质量实际上成为各类智能算法发挥效益的 瓶颈. 文献报道或尚未公布的海量实验数据是构建高质量数据集的宝库, 但长期以来缺乏用于存储、整理、产生 有关数据集的专门数据库或数据管理系统. 本文以高温永磁合金代表体系 Sm-Co基多元合金为例, 介绍了本课题 组构建的集成数据采集、数据标注、数据抽取与转换的一体化智能数据库及其管理系统. 对材料的元素成分、 物相组成、晶体结构、制备工艺、性能及其测试方法等各方面的数据建立了关系模型, 实现了相关数据的逻辑 关联并高度结构化, 从而可根据具体的材料设计需求检索出高质量的数据集. 建立的信息管理系统可实现多用户 在线标注数据, 具有数据录入准确高效、数据统一规范、最大程度消除圥余信息等特点. 应用实践表明, 建立的 专用数据库及信息管理系统在数据驱动材料设计研究领域可发挥重要作用.

关键词＼cjkstart材料数据库,信息管理系统, 材料基因工程, 数据驱动材料设计

\section{1 引言}

\section{1 材料设计用数据集的分类}

近年来数据驱动的材料设计方法在许多材料体系 的研究开发中起到了显著的推动作用 ${ }^{[1,2]}$, 而取得该类 突破的前提是构建出高质量的数据集 ${ }^{[3]}$. 数据集来源 可以分为两大类，一种来自高通量计算 ${ }^{[4]}$ 或高通量实 验 $^{[5]}$, 另一种来自己经发表的研究文献 ${ }^{[6,7]}$. 前者产生 于高通量实验装置中的传感器或高通量计算程序，其 数据采集方式属于进程间通讯 ${ }^{[8]}$ ，具有高度自动化的
特点 ${ }^{[8]}$. 高通量数据具有天然一致的数据结构, 能够方 便地导出进入目前有关数据分析软件中进行材料设 计. 然而, 此类数据直接受限于高通量实验工具或高通 量计算方法的发展状况, 因此能解决的材料科学问题 范围较窄, 例如, 统计表明近年来此类数据较大比例 集中于有关带隙的研究 ${ }^{[9 \sim 11]}$. 与高通量数据集不同, 从 分散的研究文献中获取数据集, 能充分利用前人研究 过程中产生的海量数据, 可面向现有全面的科学问题 范围 ${ }^{[12,13]}$. 借助主动学习等算法, 从研究文献构造的 一些小型的数据集也可能产生对新材料设计的巨大加

引用格式: 宋晓艳, 刘东, 刘雪梅, 等. 面向数据驱动材料设计的专用数据库及信息管理系统. 中国科学: 技术科学, 2020, 50: 786-800 Song X Y, Liu D, Liu X M, et al. Development of database and information management system for data-driven materials design (in Chinese). Sci Sin Tech, 2020, 50: 786-800, doi: 10.1360/SST-2019-0375 
速作用 ${ }^{[14,15]}$. 然而, 如何从分散的研究文献中构造产生 数据集是关键的科学问题, 这个过程面临着两大挑战: 首先, 文献中的数据结构不统一, 研究人员记录数据的 方式千差万别, 没有制订的标准、缺乏统一的数据模 型来实现这些数据的融合; 其次，对来自文献的数据 的整理过程极其繁琐耗时，且难以避免错误的产生， 函待研究开发有效工具来辅助完成.

\section{2 分散实验数据的存在形式}

大量材料实验具有过程因素多、周期长、测试表 征昂贵等特点, 与开展一次新的实验相比, 研究人员更 倾向于从已报道的文献中查阅有关实验数据. 现有文 献中蕴藏着前人开展相关材料研究积累的海量实验数 据, 例如, 本文示例的 Sm-Co 基高温永磁合金体系已经 有半个世纪的研究历史 ${ }^{[16]}$. 对材料实验而言, 研究者 通常从一次实验设计开始，包括原材料的准备、工艺 流程的设计、测试方法的制定等, 如图1所示. 根据这 样的实验方案, 研究人员首先将所需的材料样品逐个 或批量制备出来, 然后经测试分析得到一批实验结果. 最后, 根据科学问题的需要从中选择相关数据, 将科学 发现或发明创造描述到多种形式的文献中. 一方面, 这 些文献再被一些大型的文献数据库 ${ }^{[17]}$ 收录, 供人们检 索. 有时研究人员从不同的角度对这些数据进行分析, 可以得到对应不同主题的研究结果，因而有些数据被 多篇文献重复使用. 另一方面，部分测试结果符合某 一专题数据库的收录标准, 例如, 材料性能数据 库 $^{[18]}$ 、晶体结构数据库 ${ }^{[19]}$ 、相图与热力学数据

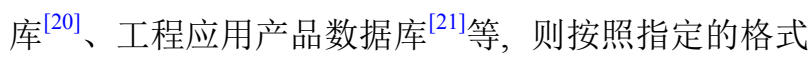
整理收录到这些数据库中, 可用于一些具体的应用程 序开发, 如物相分析、热力学计算、晶体结构精修、 产品性能比较等. 这些数据库收录的数据通常来自材 料测试表征的某一方面, 却往往丢失了与所要开发的 应用程序关系不大的其他信息，如材料制备工艺、测 试方法或其他方面的表征分析结果等.

材料设计实属逆向过程, 要实现数据驱动, 这些已 有数据中关于材料来源的信息至关重要. 尽管专题材 料数据库的庞大数据量可以借助算法开展一些研 究 ${ }^{[22]}$, 但终究还是受数据收录范围的限制. 若要达到 直接驱动材料设计的目标，材料基因工程或材料信息 学所需的材料数据更为全面, 需涵盖一批实验中的全 链条的信息. 困难的是, 除了正式发表的文献, 研究者
持有的系统详细的实验数据极少公布. 因此, 为了构建 适用于数据驱动材料设计的专用数据库, 我们经过系 列探索研究, 建立了对于文献数据的逆向追溯方法, 实现了对文献中材料数据的系统标注, 由此, 可以从 海量的分散资料中根据有关线索, 还原出已有研究中 对于某种材料的小型实验数据集，进而通过建立统一 的数据模型将这些数据有机组织起来.

2018年国务院办公厅发布了《科学数据管理办 法》, 提出了“法人单位及科学数据生产者要按照相关 标准规范组织开展科学数据采集生产和加工整理, 形 成便于使用的数据库或数据集”的要求. 将来, 随着数 据模型和相关信息管理系统逐渐发展成熟, 研究者就 能够方便快捷地将持有的小型实验数据集加入到某些 大型数据库中, 形成对实验中材料样品信息的全方位

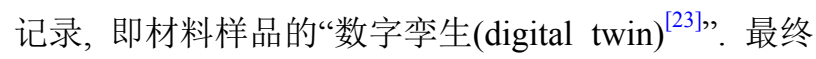
让每个小型的实验数据集都能放在更广泛的材料数据 中利用不断改进的智能算法持续开展研究.

\section{3 分散实验数据管理现状及面临的问题}

对于较简单的数据结构, 研究人员通常利用 Microsoft Excel等软件来辅助整理材料数据, 也可以 使用商业化的数据管理软件如Navicat, SQLyog和开源 的数据库管理软件phpMyAdmin等直接在数据库的关 系表上输入数据, 进行数据库内容建设. 这种方式适合 “所见即所得”的数据, 即表格数量较少, 表间关联较 少, 易于在表格中看清一条数据的全貌.

随着材料数据库应用的拓展，尤其是面向材料基 因工程的需求，其数据结构需根据材料学数据之间的 本质关联进行设计，从而能够适应不同方面的需求和 应用中数据的组织形式，这种结构并非“所见即所得”, 而是由大量的 “关系”连接形成的庞大而复杂的数据体 系. 为了应对在这种复杂数据结构下的数据管理工作, 需要建立信息管理系统这种行之有效的方法. 信息管 理系统已经在金融、会计、文献管理等领域得到普遍 应用, 也加速了这些领域的数字化和智能化发展进程. 针对材料基因工程专用数据的管理软件尚处于起步阶 段, 近年来一些大型材料数据平台已着手开发数据管 理工具, 如美国国家标准与技术局开发的材料数据管 理系统(Materials Data Curation System, MDCS) ${ }^{[24]}$, 该 系统用XML文件定义数据模型, 根据该模型生成数据 采集表单. 由于材料数据复杂异构的特点, 这种由大型 

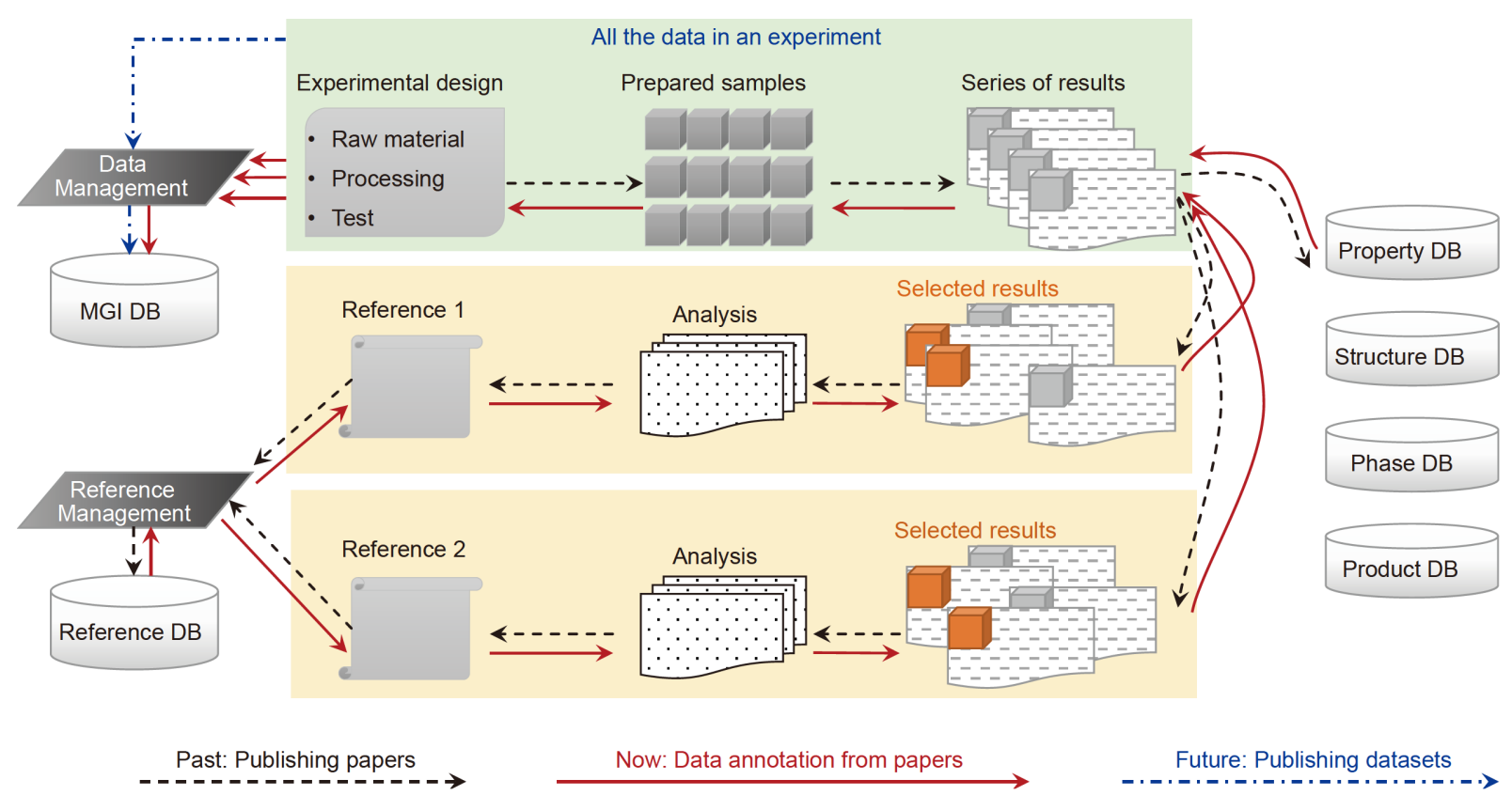

图 1 (网络版彩图)材料实验数据在过去的产生与发布流程、目前面向材料基因工程的数据库建设方式与数据驱动材料设计 专用数据库的发展趋势

Figure 1 (Color online) The generation and publication processes of materials' experimental data, the construction frame of databases for Materials Genome Initiative applications, and the development trend of materials databases for data-driven materials design.

平台提供的管理系统致力于在满足高度通用性的基础 上, 为数据采集工作提供易懂的表单页面, 但却缺失功 能模块来同时实现便捷性与规范性. 实际上, 材料数据 存在大量的关联和约束条件, 例如, 在研究人员持有的 小型数据集中，不同的材料数据在测试方法上可具有 高度一致性，在制备工艺过程方面可具有高度关联性， 在单位制、物相、工艺参数等方面又可能具有大量的 约束条件. 现有的数据管理工具很难帮助人们对这类 数据建立关联，在这类数据的整理过程中不得不借助 大量的人力来重复录入或梳理有关信息，由此得到的 数据集也很难进一步获得广泛应用.

如上所述，数据驱动的材料设计依赖于高质量的 数据集，而高质量数据集的缺乏是当前发展的主要瓶 颈. 从分散的研究文献中构造数据集是目前一条重要 的可行途径，然而却面临着如下两方面的问题：一是 文献中数据产生于过去，分布于前人发表的文献或部 分收集于专题数据库中，数据的有效集成难度很大; 二是目前缺乏可辅助采集复杂异构的材料数据的信息 管理系统, 为最终获得高质量的数据集急需提高采 集、管理和应用数据的效率.

在这样的背景下，本课题组在大量探索研究的基
础上, 提出了一整套的数据治理方案. 首先, 建立了面 向材料基因工程应用的数据关系模型，集成了材料成 分与物相组成、晶体结构、制备工艺、性能等多方面 数据, 建立了数据项之间的科学逻辑关联, 数据的规范 性、可读性和可重复利用性显著提高. 然后, 开发了材 料知识信息解析、关联与管理系统，使研究人员能够 对文献进行准确的数据标注, 且最大程度消除了几余 信息，保证数据质量的同时大幅度提高了数据采集效 率. 在此基础上, 围绕数据驱动材料设计的目标, 实现 了传统数据库难以突破的材料深层次信息检索、材料 数据库融合汇交、高质量材料设计数据集抽取等功 能. 本文依托 $\mathrm{Sm}-\mathrm{Co}$ 基多元合金这种典型的高温永磁 合金代表性体系，介绍本课题组构建的面向数据驱动 材料设计的专用数据库及相应的信息管理系统，在示 例其应用的基础上，讨论并展望这类专用数据库及相 关知识信息系统的发展前景.

\section{2 面向数据驱动材料设计的专用数据库建 设方案}

图2所示为本文设计的面向数据驱动材料设计的 


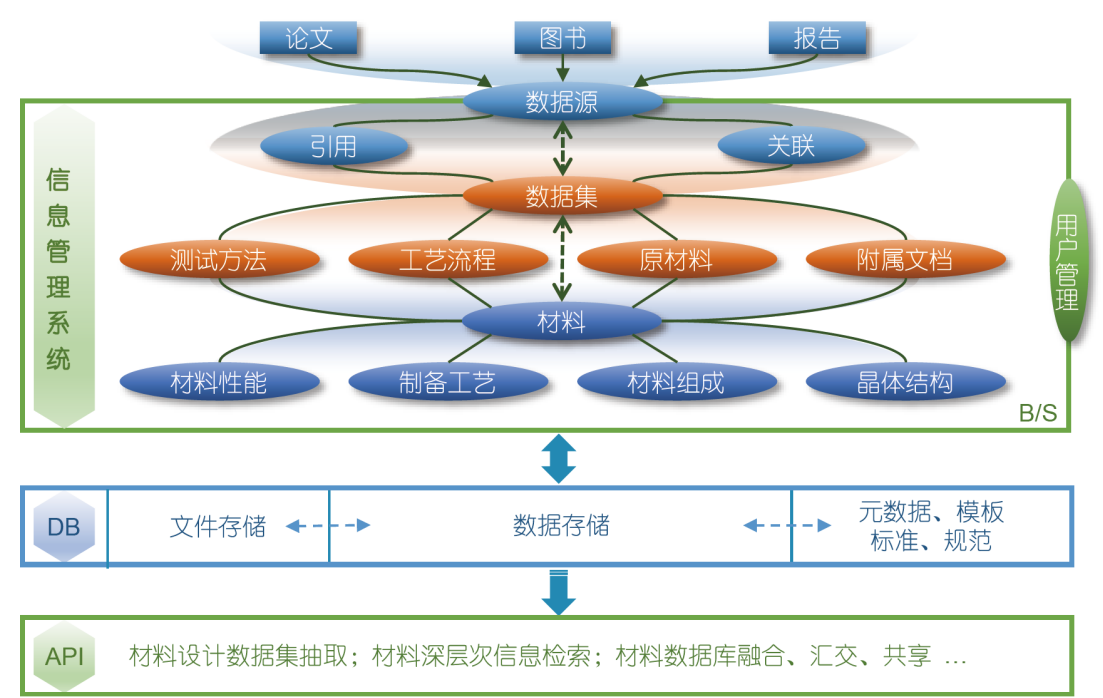

图 2 (网络版彩图)面向数据驱动材料设计的合金类材料数据库建设方案

Figure 2 (Color online) Construction layout of alloys database for data-driven materials design.

合金类材料数据库建设方案，主要包括数据库建模、 信息管理系统开发和数据库应用三个方面，在这三方 面的基础上开展数据库内容建设.

$\mathrm{Sm}-\mathrm{Co}$ 体系数据是高温永磁合金领域数据驱动新 材料设计的基础, 为了使数据库应用满足数据检索、 抽取和汇交等技术的要求, 对 $\mathrm{Sm}-\mathrm{Co}$ 体系专用数据库 的建设采用数据逻辑更加清晰的关系型架构. $\mathrm{Sm}-\mathrm{Co}$ 体系数据在单位制、制备工艺等方面存在复杂异构的 特点, 无法用传统的数据标注方式进行数据采集工作, 而信息管理系统则是解决这一难题的关键工具. 本研 究建立的信息管理系统将为研究人员开展数据标注工 作提供便捷的环境，同时具有数据采集、多用户协 作、数据导入、数据处理、数据标注、数据可视化等 多种功能. 系统架构采用 $\mathrm{B} / \mathrm{S}$ 模式，使用者可在不同的 操作系统登陆并应用到最新上线的功能. 利用DRY (don't repeat yourself)思想 ${ }^{[25]}$, 使研究人员避免重复录 入相同的信息, 从而大大减轻数据标注工作量, 并保证 相关数据的规范统一.

按照被管理的对象，将信息管理系统划分为数据 源、数据集、材料三个层次. 数据源指不同渠道的论 文、图书、报告等，是一种离散型数据文本. 数据集 是在一批实验中材料样品共有信息的组织模块，如原 材料、制备工艺、测试方法、性能、文献发表信息 等, 对应以前研究人员的一套实验方案. 这些共有信息 通过管理系统进行自动填充并经系统管理员审核后成
为一项具体材料样品的专有信息. 建立的该数据库的 应用, 一方面, 可开发同类合金的数据共享网, 与传统 的数据共享相比, 可实现数据在多维度检索层次上的 深度信息共享; 另一方面, 利用抽取-转换-导入技术 (extract-transform-load, ETL) 获取数据集, 进行数据驱 动的材料设计. 此外, 通过开发应用程序接口(application programming interface, API), 可以对接数据分析软 件或面向材料基因工程应用的数据库汇交平台.

\section{3 面向数据驱动材料设计的合金类数据关 系模型}

\section{1 数据源与材料数据的关系建模}

在图1所示的流程中, 从数据源还原产生可用于数 据驱动材料设计的专用数据的过程, 是前人从实验数 据产生论文等数据源的逆向过程. 前面研究人员持有 的小型数据集是数据源与材料数据之间关系的关键实 体. 小型数据集与材料样品是一对多的关系, 材料数据 在这样的小型数据集中通常格式统一, 在后续数据库 及信息管理系统的开发中对该特点加以利用, 可有效 加快数据标注和数据处理工作. 对于一些期刊论文, 通常数据源与小型数据集是多对一的关系, 而对于图 书或学位论文等篇幅较长、信息更丰富的文献, 数据 源与数据集存在多对多的关系, 但数据集的数量远小 于材料样品的数量. 因此, 通过对数据源中的内容进 
行梳理, 先还原产生研究人员曾持有的小型数据集, 再 通过整合数据集完成数据驱动材料设计专用数据库的 构建，是一种比直接从数据源抓取材料数据更便捷、 准确的数据库建设方式. 根据上述思路建立的数据源 相关信息的关系模型示例如图 3, 其中MySQL数据库 的关系表用IDEF1X方法表示. 文献, 即数据源(命名为 reference), 这个实体具有人们熟知的多种属性, 包括题 目、DOI、作者、出版年、文献类型等.一个数据集 (dataset)对应着多种材料样品(material), 其中一部分材 料样品是以原材料(raw)形式存在的; 原材料具有纯 度、规格、生产商、生产批次等信息，同一数据集中 的其他材料均制备来自这些原材料。该关系模型中的 数据集对应一批实验，在这批实验中，针对某类材料 性能(property_define), 存在若干既定的测试方法(measurement). 因此，测试方法与数据集是多对一的关系, 与材料性能也是多对一的关系.

\section{2 材料制备工艺关系的建模}

在文献中材料的制备工艺常以一段文字说明的形 式呈现在数据源的“制备方法”部分，包含工艺流程、步 骤名称、工艺参数以及材料的转变过程等信息, 原则上 研究人员通过阅读该文本可重复实现对该合金的制备.
然而, 这种文本形式的工艺数据是非结构化的, 不具备 “可发现”、“可获取”、“可交互”等科学数据的特点 ${ }^{[26]}$.

Sm-Co基合金的制备过程通常由多步工艺构成， 这类信息可采用流程图表示，在一批实验中往往存在 多种中间产物和目标产物，这些产物都有可能被检测 表征. 因此, 可以认为, 工艺数据实际上蕴含着材料样 品与材料样品之间的关系. 本研究首先通过在关系型 数据库中建立“有向无环图”(directed acyclic graph, DAG)，将这种样品之间的关系结构化. 在此基础上扩 展其他数据, 实现所有工艺信息的结构化. 图4示出了 相关部分在一个工艺步骤中涉及信息的逻辑关系。该 步骤中，原料与步骤产物都是DAG中的“结点”(node), 二者之间的关系即DAG的“连接”(link). 其余工艺步骤 中的数据, 则建立在这个结点和连接的结构之上: 一个 工艺步骤包含一组连接，具有工艺名称和工艺类型等 属性，而工艺参数是与工艺类型有关的一个数据表， 与此组连接对应，而其他材料数据则对应着每个具体 的结点.

在关系型数据库中可以采用邻接矩阵或邻接表的 方式展示DAG，如图5所示. DAG中的连接，用邻接表 (processing_link)表示, 该表的三个主键均为外键, 第一 个表示本步工艺使用的起始材料(InputMaterialID)，第

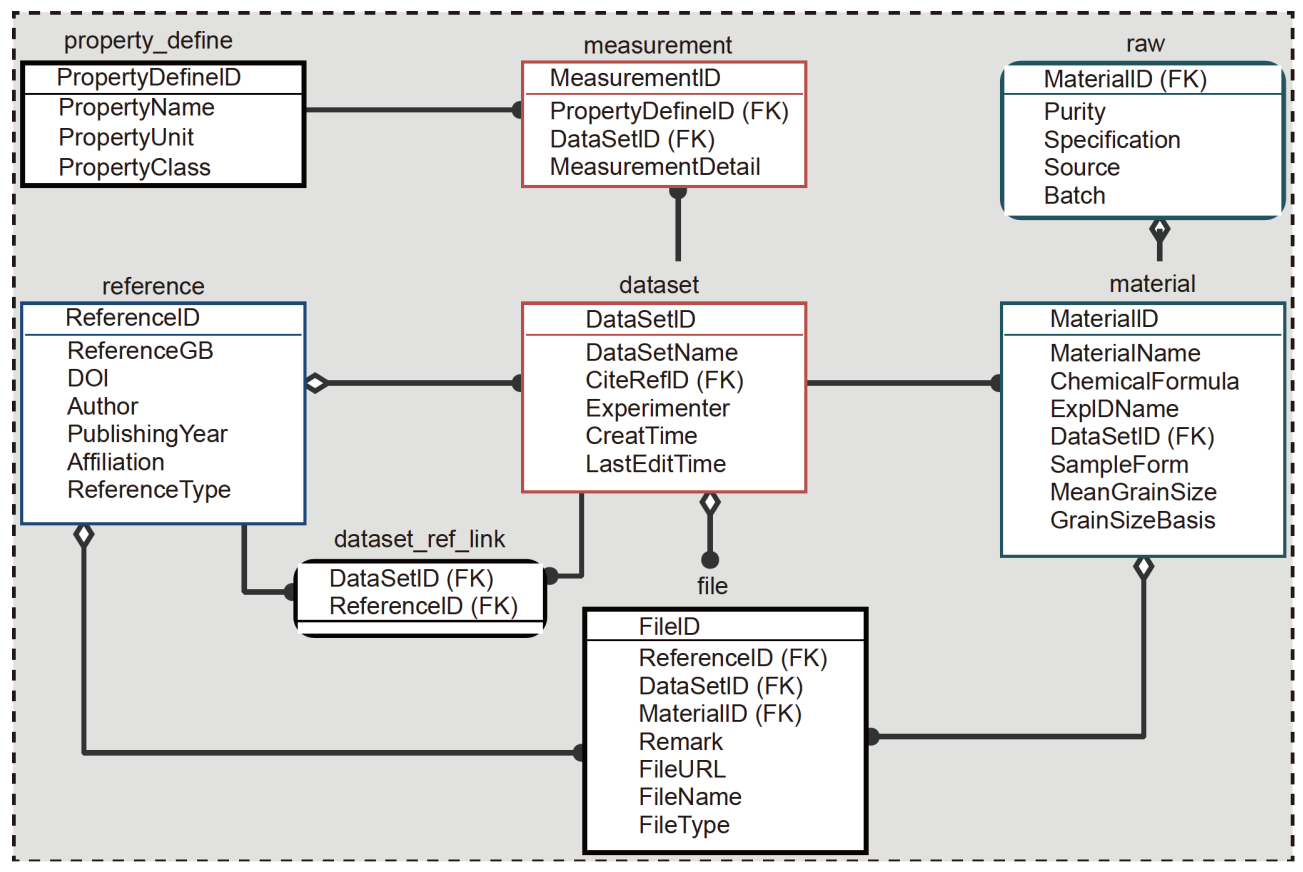

图 3 (网络版彩图)数据源相关信息的关系模型

Figure 3 (Color online) Relational model for the information from the data source. 


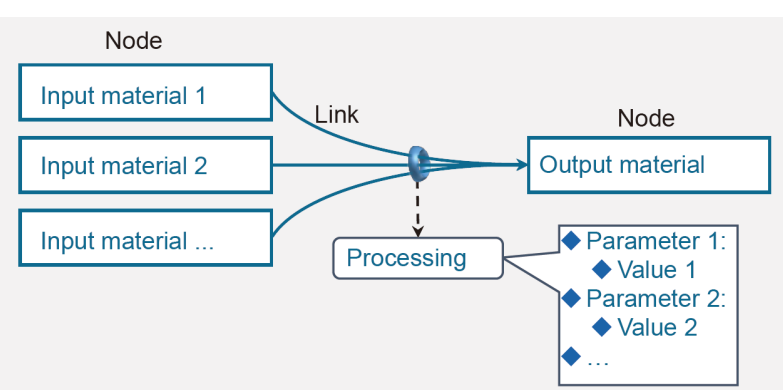

图 4 (网络版彩图)制备工艺中任意一个步骤信息的图形结 构化表示

Figure 4 (Color online) Structured representation of the information in one step of the preparation process

二个表示本步工艺得到的阶段性产物(OutputMaterialID), 第三个表示从起始材料到阶段性产物这一过程所 属的工艺步骤(ProcessingID). 同时, 也可以相应记录各 起始材料的使用量(InputMaterialContent). 工艺表(processing)用于收录所有工艺类型共有的数据属性，如工
艺名称(ProcessingName)、工艺类型(ProcessingType)、工艺叙述性文本(ProcessingDescribe)等.

工艺具有大量的子类，子类的数据结构各不相同， 如图5(a)所示, 在processing表中的工艺类型(ProcessingType)可以进行类别鉴定，根据其取值选择对应的 工艺类型表，如放电等离子烧结参数表sps、球磨参数 表ball_milling、退火参数表annealing等. 当然，图5(a) 所示并非涵盖完全的类别, 随着制备工艺的拓展, 可建 立更多的工艺参数表与之对应. 本研究将这种可不断 扩展的工艺参数的元数据信息存储在工艺定义表(processing_define)中, 如图5(b)所示, 工艺类型(ProcessingType)和工艺参数名称(ParameterName)为其组合主键. 因此, 一个工艺类型与多个参数名称对应. 工艺参数的 数据存储在processing_detail表中, 该表有三个约束: 一 是该数据属于一个工艺步骤(ProcessingID); 二是该数 据符合该工艺步骤给定的工艺类型(ProcessingType);
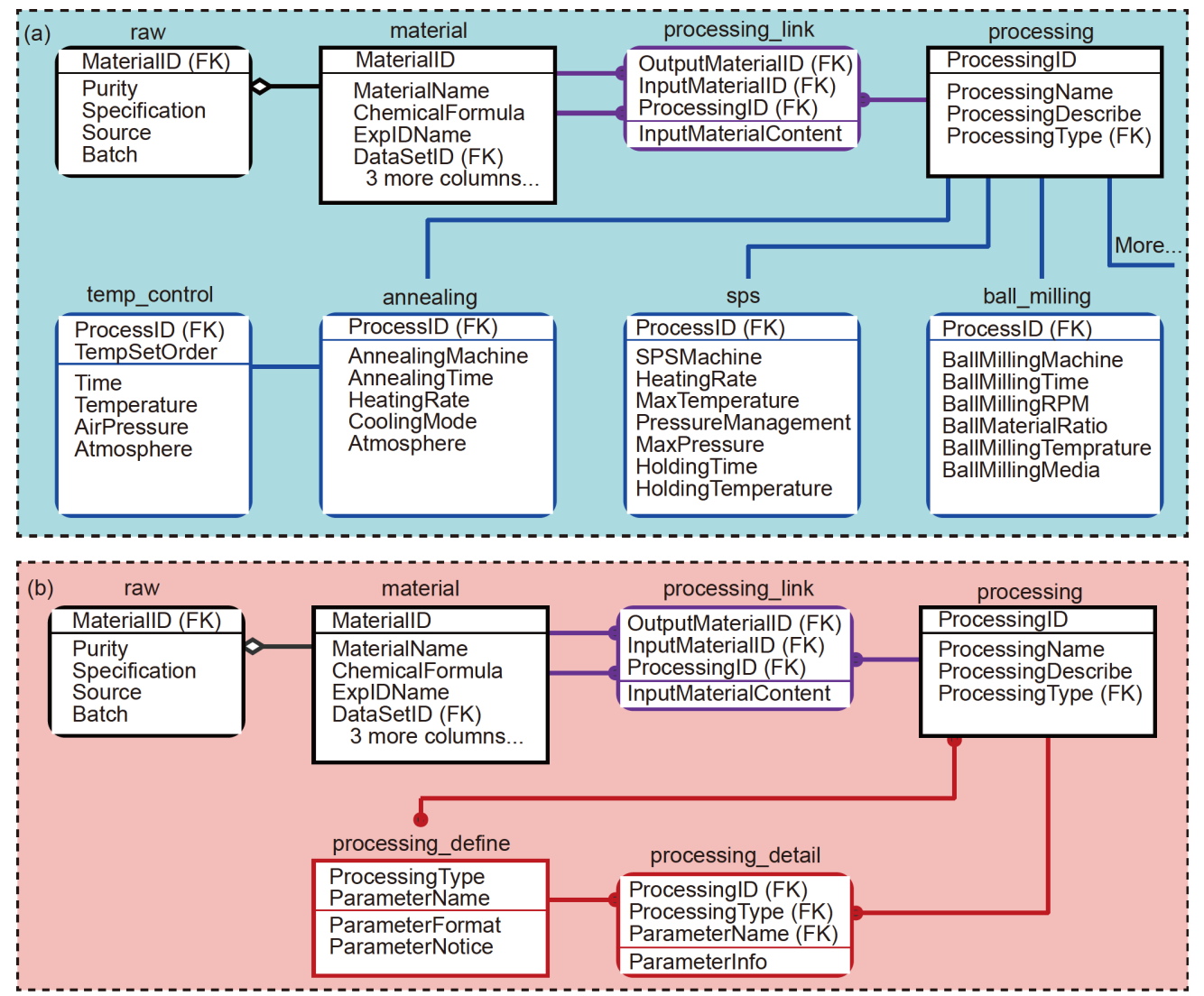

图 5 (网络版彩图) 以 Sm-Co基合金为例建立的制备工艺相关数据关系模型. (a) 使用独立的工艺参数表; (b) 利用元数据模板 化的工艺参数表

Figure 5 (Color online) The established relational model for processing information using Sm-Co based alloys as an example. (a) Independent parameters table; (b) templated parameters table using metadata. 
三是该数据对应该工艺类型下设定的参数名称(ProcessingName). 在数据标注中, 如有新的工艺参数出现, 在工艺定义表中更改记录即可改变工艺参数的记录范 围, 无需在关系模型中增加新的数据结构, 也无需在相 应的数据库应用软件中调整接口程序. 经大量的数据 收录过程证明，本研究建立的这种工艺关系模型能够 灵活和准确地应对多元合金工艺种类和工艺参数的扩 展和补充.

\section{3 材料性能数据的建模}

对永磁材料而言，其性能指标体系复杂、单位制 多样. 除材料性能的名称、单位与取值外，性能数据 还包括测试方法、条件等，可幸的是，在一批实验中， 针对一类具体的材料性能通常采用相同的测试方法. 尽管性能数据复杂多样，但相关领域的研究人员常有 共识，即对性能数据常存在被公众认可的规范或标准. 可知，材料性能、测试方法和性能规范之间是相互依 赖的关系. 本研究建立的性能相关数据的关系模型如 图6所示，性能规范被定义在表property_define中，包 括性能的名称、单位制、种类等. 表property用于存储 材料的性能, 其数据既属于某一个具体的材料样品, 又 符合指定的性能种类, 因此包含两个外键. 一些测试条 件, 如温度, 可将其作为性能的一个属性. 文献中测试 方法相关的信息被整理到表measurement中，这些测试
方法就是针对同一批次实验中材料性能的通用测试方 法. 性能的同义词或非标准的命名方式均被收录在表 synonym中，与表property_define的某一规范的分类名 称相对应. 性能数据之间的单位转换通过 transformation实现，其主键包含两个外键，一个指明当前存储的 材料性能类型(CurrentDefineID), 另一个指明作为转换 目标的材料性能类型(TargetDefineID), 并记录与二者 对应的单位转换脚本(ConversionScriptID).

\section{4 材料元素、物相、晶体结构等数据的建模}

元素、物相、组织等数据具有类似的特点, 它们 都是按照某一规则对材料样品进行划分的结果，与材 料样品均为多对一的关系. 名称和含量是其共有的基 本属性, 同时也具有各自特殊的属性, 例如, 物相在粉 末衍射数据库中对应PDF(powder diffraction file)编号, 元素对应有原子比和质量比等. 元素与其性质之间是 确定的一对一关系，每一个元素有与之对应的一条性 质数据，如电负性、原子半径、价电子等. 人们对物 相的命名尚未形成标准，在不同的文献中存在大量的 同义词现象，本研究中将最常用的命名方式作为默认 名称，以保证数据的可发现性。一种物相对应着一种 原子排布结构，如果该物相是结晶相，则对应着一种 晶体结构, 二者是一对一或一对零的关系.

根据以上分析，建立的材料元素、物相、晶体结

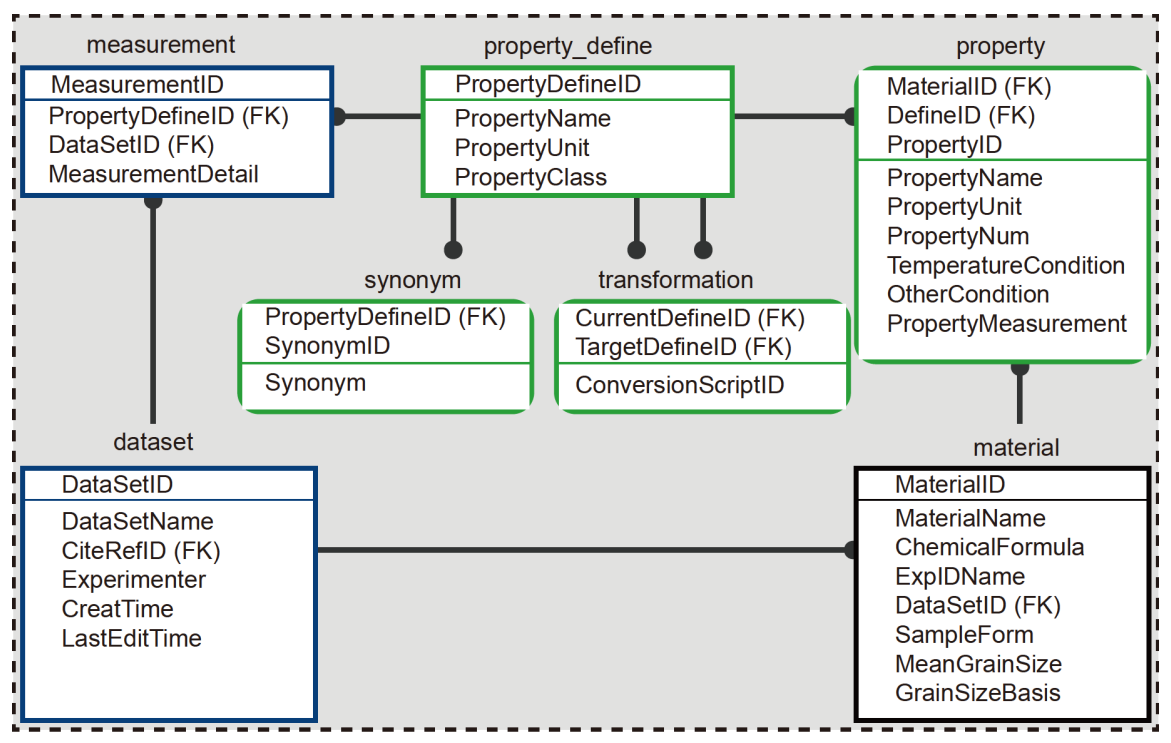

图 6 (网络版彩图)材料性能相关数据的关系模型

Figure 6 (Color online) Relational model of properties related data of materials. 
构等数据关系模型如图7所示. 存储材料元素、物相和 其他(如组织)数据的关系表中分别设置了与材料样品 对应的外键, 形成组合主键. 存储材料晶体结构信息的 表通过外键与其对应的物相数据建立关系. 晶体结构 数据包括晶体结构类型、空间群符号和序号、晶体点 阵参数、晶体学信息等. 材料构成元素的大量特征存 储在element中，既约束着元素数据的名称，也用于提 供数据挖掘时合金构成元素的特征.

将上述数据源、材料元素及物相组成、晶体结 构、制备工艺、性能等多个方面的关系模型集成后， 来自分散数据源的材料数据可准确地存储在数据库的 各个关系表中，充分满足对科学数据的可发现、可获 取、可交互、可再利用(findable, accessible, interoperable, reusable, FAIR) 要求 ${ }^{[26]}$, 而且数据项之间紧密关 联, 数据体系高度结构化, 可快速检索到规范格式的各 类数据.

\section{4 材料知识信息解析、关联与管理系统}

图8示出本研究建立的材料知识信息解析、关联 与管理系统的主要模块. 其中, 用于数据管理的核心模 块是文献管理模块和数据集管理模块. 文献管理模块 处理具体领域相关的期刊论文、学位论文、研究报告
等需要解析的分散数据源, 其功能包括文献的查阅与 管理、文献的导入与重复收录检查、文献信息的编辑 等. 数据集管理模块用于处理由一批实验中的材料数 据构成的数据集, 包括数据集的查阅与收藏、新数据 集的创建及数据集包含信息的编辑等. 利用这两个模 块可以将文献归类、汇总到每个与之有关的小型实验 数据集中.

当来自一批实验的相关文献收集完成后，即可开 展数据集公共信息的创建，即数据集编辑模块. 系统 通过对数据进行实时可视化让使用者及时了解数据集 的收录状况，并通过对比两个数据集中材料的成分、 工艺等信息及时识别可能重复收录的数据集. 数据驱 动材料设计的专用数据可在每个数据集的材料信息编 辑模块进行管理，在此可调整具体材料的工艺细节、 录入材料的组成信息(包括成分、物相、组织等)以及 填写材料的性能数据和编辑晶体结构信息等. 在上述 高度模块化的系统中, 可以准确、高效地开展数据采 集工作(流程参见图2和8).

在一批实验中, 以其中任意一个材料样品为起点, 对工艺关系的邻接表进行迭代查询, 即可得到每种材 料的前序或后序材料，均能遍历这批实验所有的材料 样品信息, 示例如图9. 该图为根据检索获得的材料样 品之间的工艺关系生成的工艺流程图，可见所有的材

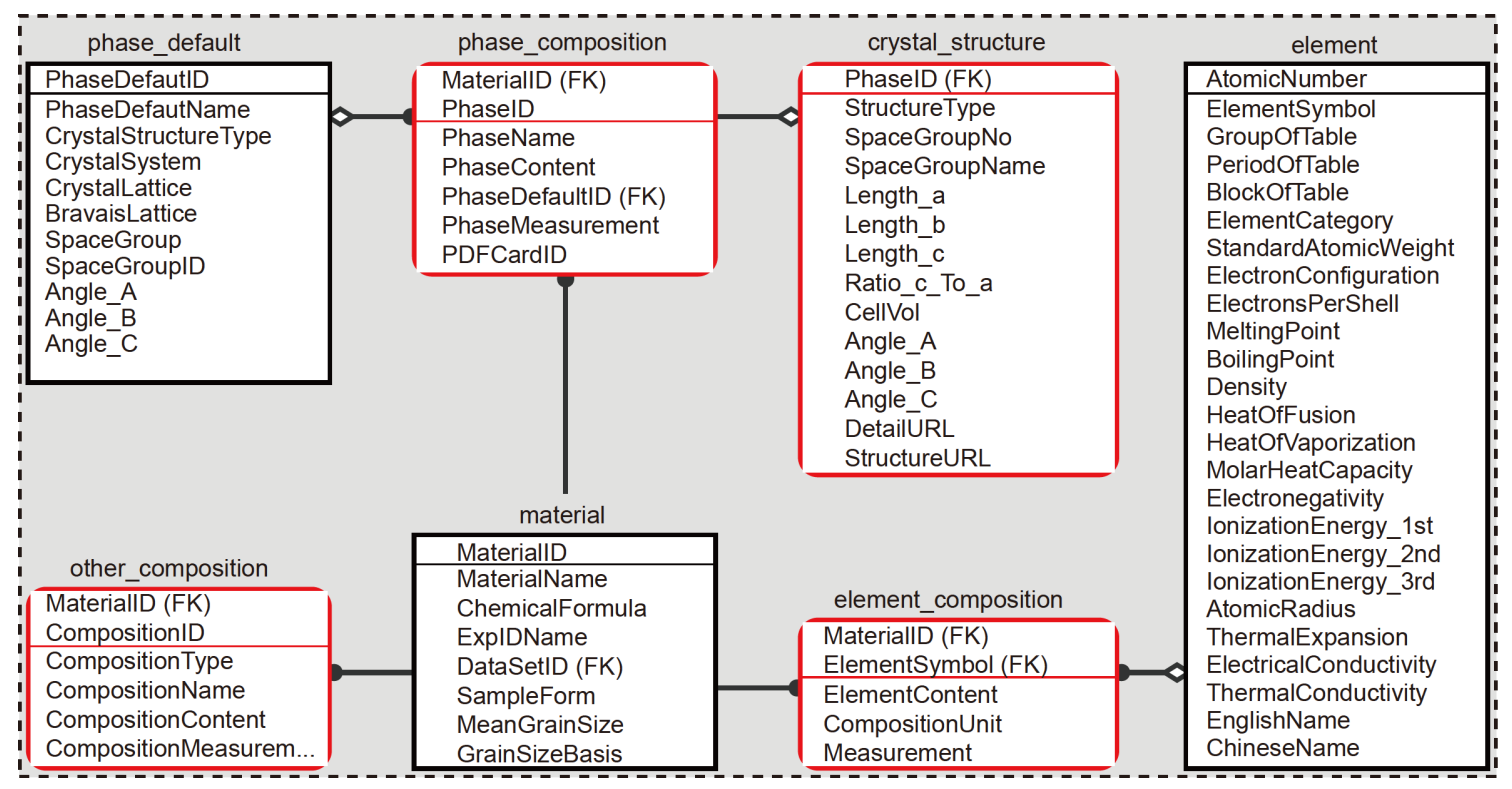

图 7 (网络版彩图)材料元素、物相、晶体结构及其他相关数据的关系模型

Figure 7 (Color online) Relational model of elemental composition, phase constitution, crystal structure and other related data of materials. 


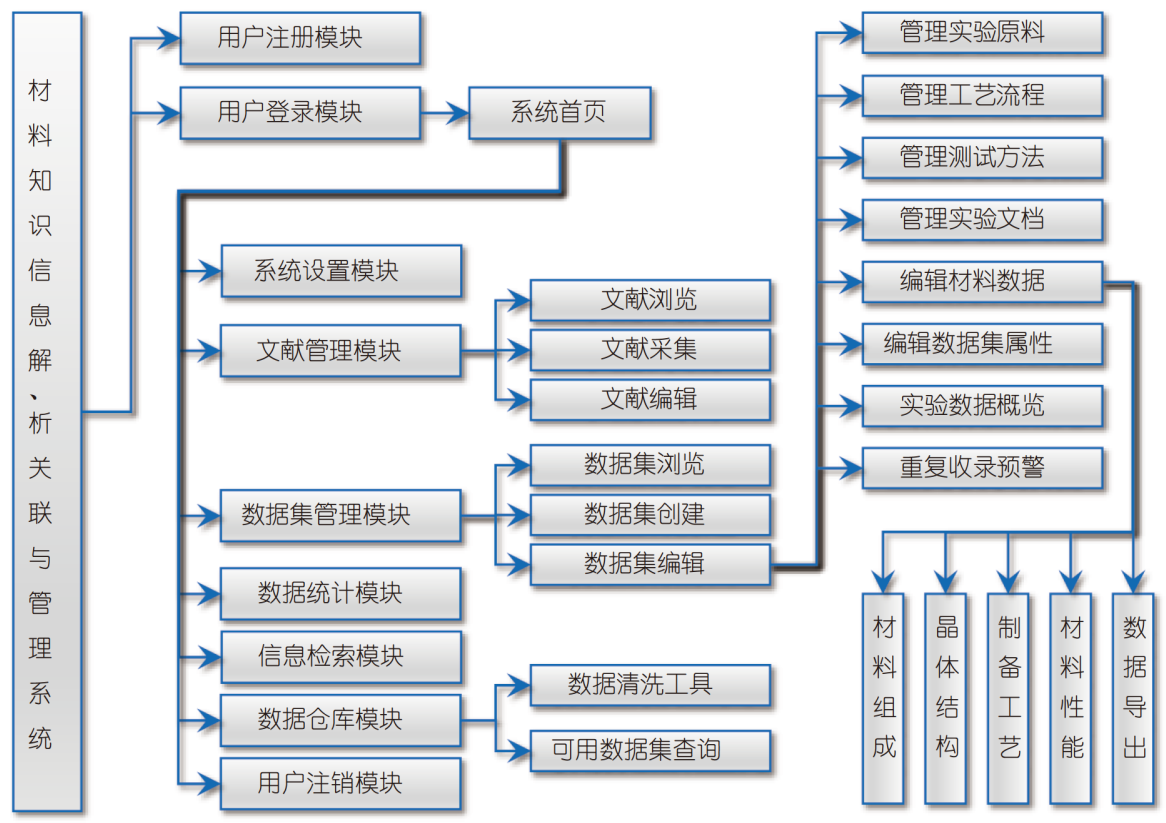

图 8 (网络版彩图)面向数据驱动材料设计的合金类材料数据的信息管理系统主要模块

Figure 8 (Color online) Main modules of the information management system for the data available for data-driven materials design.

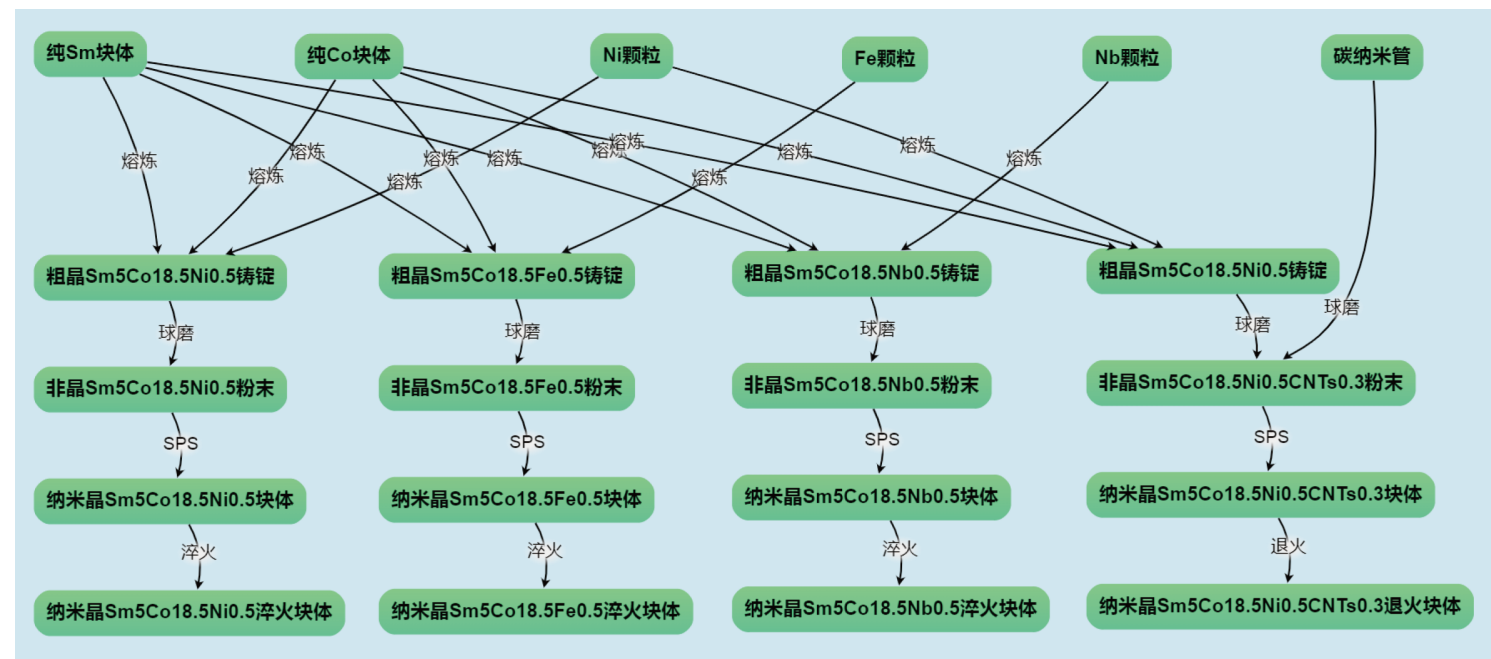

图 9 (网络版彩图)在系统中根据检索获得的材料样品之间的工艺关系生成的工艺流程图

Figure 9 (Color online) Process flow diagram generated in the information management system based on the retrieved relationship between processing of material samples.

料样品信息由工艺流程连接起来，沿着每个材料样品 的制备流程可逐步查询，向上最终可追溯至原材料信 息. 由此可以看出，本研究建立的以还原产生一批实 验中原来形成的小型数据集为媒介的方法，解决了传 统材料数据库中难以对工艺数据进行结构化的难题.

图10以树状图形式示例了 Sm-Co体系专用数据库
中性能数据的存储状态. 依次按数据集、材料样品、 性能种类划分为不同的区块，区块面积表示该分类下 的相对数据量. 白色边界间隔开的代表数据库中存储 的不同数据集，其面积表示一批实验数据中收录的材 料性能数据总量, 可见不同数据集的数据量是不均衡 的. 在每个数据集中，进一步由灰色边界区分出不同 


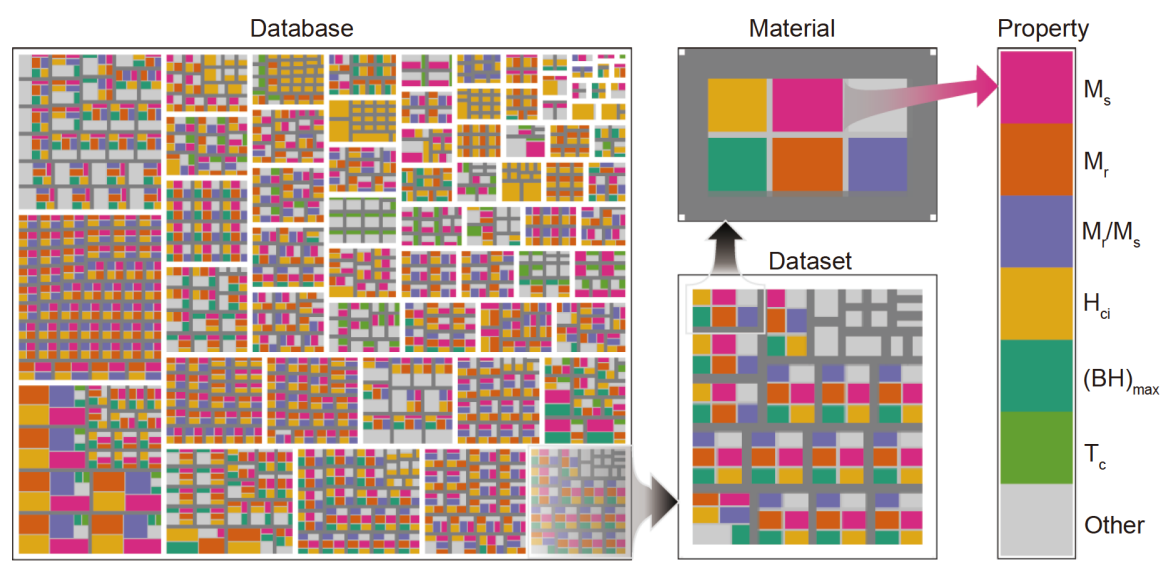

图 10 (网络版彩图)系统读取专用数据库并依次按数据集、材料样品、性能种类生成的性能数据量树状图(以Sm-Co基高温 永磁合金体系为例)

Figure10 (Color online) The treemap of properties data volume generated by the information management system after querying the database and grouping the data according to dataset, material sample, and property category (using Sm-Co permanent magnetic alloy system as an example).

的材料样品, 每种材料样品的不同性能数据量由色块 大小表示. 从各个数据集的色块分布可以看出, 每个 数据集聚焦的性能种类有所不同; 同一个数据集中的 各材料通常聚焦了相似的性能种类，这种现象是来自 文献的数据集的常见特征，在系统中对这些公共信息 初步梳理后, 可让研究人员在开展数据标注时聚焦到 关键数据上.

图9所示工艺数据的可视化和图 10 所示性能数据 的可视化, 均是由软件对数据库直接读取生成, 这种实 时的图像生成模式, 表明本研究构建的数据模型、材 料信息管理系统这一整套的数据管理方案完全实现了 软件或机器对数据的可读和可处理. 这一点正是开展 数据挖掘、机器学习等数据驱动的材料设计对数据库 的紧迫需求.

\section{5 专用数据库及信息管理系统的应用}

\section{1 基于深层次材料信息的数据检索}

在已有的文献数据库中, 人们通常使用字符匹配 的方式从中检索论文. 然而, 材料、化学、生物等专 业领域存在一些独特的字符编排方式, 如化学式、物 相等, 在文献数据库中, 对这些信息的识别度不高, 例 如元素In, 此元素符号同时也是英文中常用的单词, 由 此造成搜索结果的准确性很差. 因此, 人们更希望能够 从深层次的信息中进行搜索. 在本文构建的关系模型 中, 文献、数据集、材料数据与材料深层次信息均建
立了直接或间接的关联，可以利用结构化查询语言， 准确地从数据项开展文献检索、数据集检索和材料数 据检索.

图11示例了材料数据的检索页面, 在列表中显示 的是检索结果, 即材料的简要信息, 包括材料名称、 组成、工艺、性能、参考文献、实验信息等. 在数据 检索页面上方提供有4种材料信息检索方式: 元素、 物相、性能和工艺. 在“元素笁选”栏, 提供系统中收 录的所有材料的构成元素及其查询结果数目, 点击元 素名称即可对材料信息列表中的结果进行笁选, 得到 含有该元素的相关材料信息. 在 “物相篎选”栏, 提供 系统中收录的所有物相组成及其查询结果数目, 点击 物相名称即可对材料信息列表中的结果进行精炼, 得 到含有该物相的相关材料. 在 “性能篮选”栏, 给出了 系统中所有材料性能的名称及其收录数量, 点击相应 的性能名称可以将收录了该项性能的所有材料信息展 示在检索列表中. 类似地, 在“工艺笁选”栏, 给出了系 统中所有材料最后一步制备工艺的工艺名称及其查询 结果数目, 通过相应的工艺名称即可检索到来自该工 艺的材料数据.

\section{2 数据转换、融合与汇交}

本研究中数据关系模型分别围绕数据源、数据 集、材料三个主要实体进行构建, 关系模型具有高度 结构化的特征, 无论以哪种实体为根节点, 都可以从 数据库中抽取出一条完整的数据记录, 因此, 完全可 


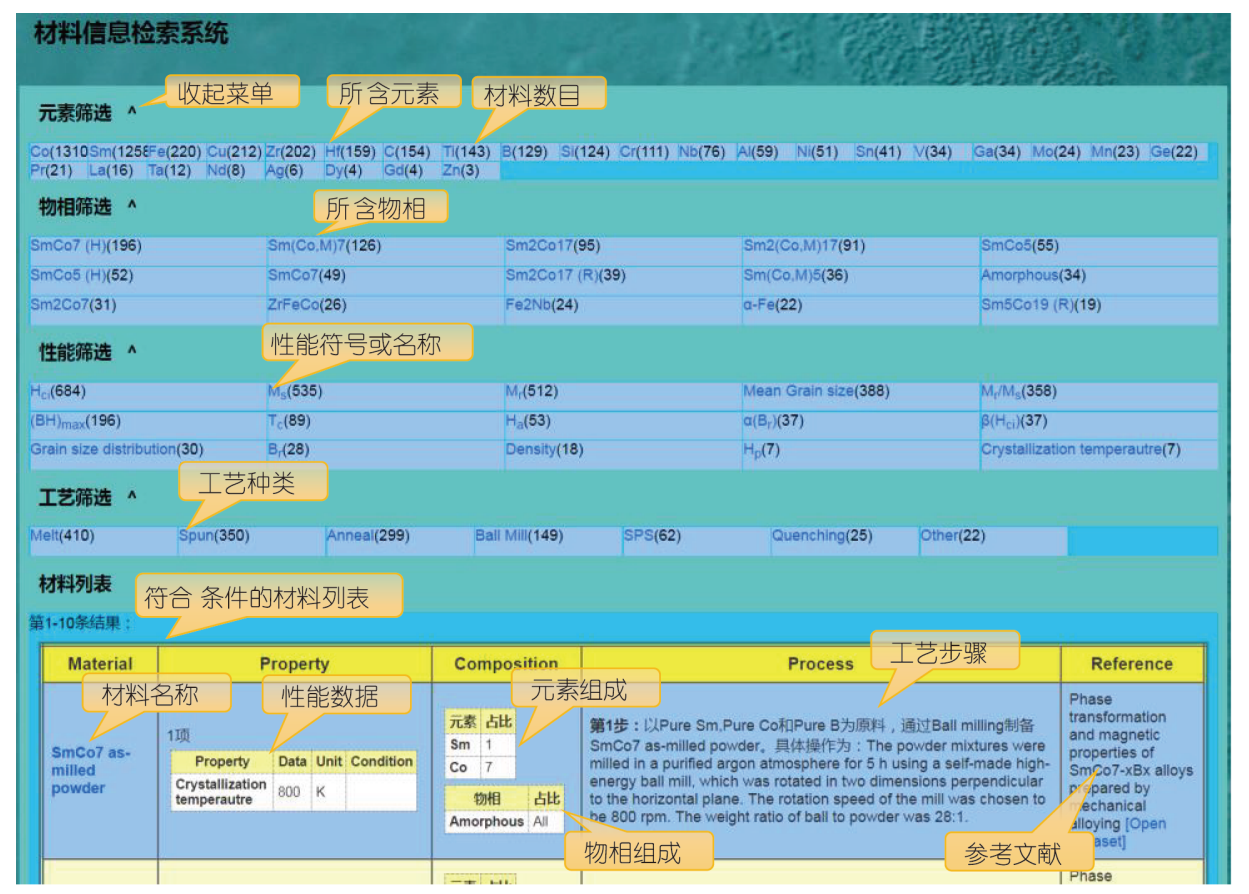

图 11 (网络版彩图)在系统中检索材料数据信息的页面示例

Figure 11 (Color online) An example page of retrieving material data and related information in the system.

以根据不同大数据平台的数据结构设计接口, 将本系 统的数据批量导入平台. 其中, 面向材料基因工程的 专用数据库以材料实际样品或虚拟样品为研究对象, 因此从关系型数据库中以材料实体为根节点, 抽取出 数据库中所有与该材料相关的数据，包括材料名称、 元素组成、物相组成、晶体结构、制备工艺、性能、 文献信息等多个方面, 由此集合形成专用数据体系. 例 如, 对于具有XML文件导入功能的材料数据共享平台, 本研究建立的系统可根据平台制定的文件格式，通过 脚本语言抽取数据库中的数据, 进而转换为包含有材 料数据信息的XML文件，如图12所示，将该文件或文 件的访问地址导入平台，即可实现有关数据从本地数 据库向数据共享平台的快速汇交. 如前所述，一条用 于数据驱动材料设计的专用数据的内容非常丰富, 在 模块化的信息管理系统的辅助下，能够高效地形成这 样全面的材料数据信息, 大大降低了数据标注的难度 并显著减少了数据标注的返工次数.

\section{3 材料设计专用数据集的构建}

在进行材料设计时，通常以材料的某项性能或某 种物相、晶体结构为目标和研究对象. 在本研究建立
的专用数据库及信息管理系统中，可以从高度结构化 的数据体系中, 通过结构化查询语言, 笁选出与目标/ 研究对象所有可能相关的数据项，汇总得到面向材料 设计的数据集. 为了能够随时从系统中掌握数据集的 状态从而针对性地补充、优化相关数据, 本研究专门 开发了机器学习可用数据集的检索模块. 根据目前机 器学习算法在材料设计中的应用状况，将一些常见的 材料性能(常用作回归学习目标变量)和材料物相(常用 作分类学习目标变量) 与材料的元素组成、工艺种 类、样品性状等特征连接查询, 在系统中即可形成大 量的可用于机器学习的数据集. 如图13所示, 数据库 中收录的数据按照元素组成和目标变量种类可划分为 若干数据集，其数字表示当前数据集中的材料种类数. 通过每个分类下的超链接即可汶览该数据集的内容, 图中示例的分别是三元合金饱和磁化强度数据集和三 元合金物相组成数据集. 在数据集构建的过程中, 若要 对错误数据进行修改、对缺失数据进行补充或进行更 多材料特征的查询, 可在系统中进行“在线数据清洗”. 本研究建立的专用数据库及信息管理系统大大加速了 高质量数据集的构建过程. 通常人们在分析软件中对 数据集进行清洗，本系统建立的这种“在线数据清洗” 


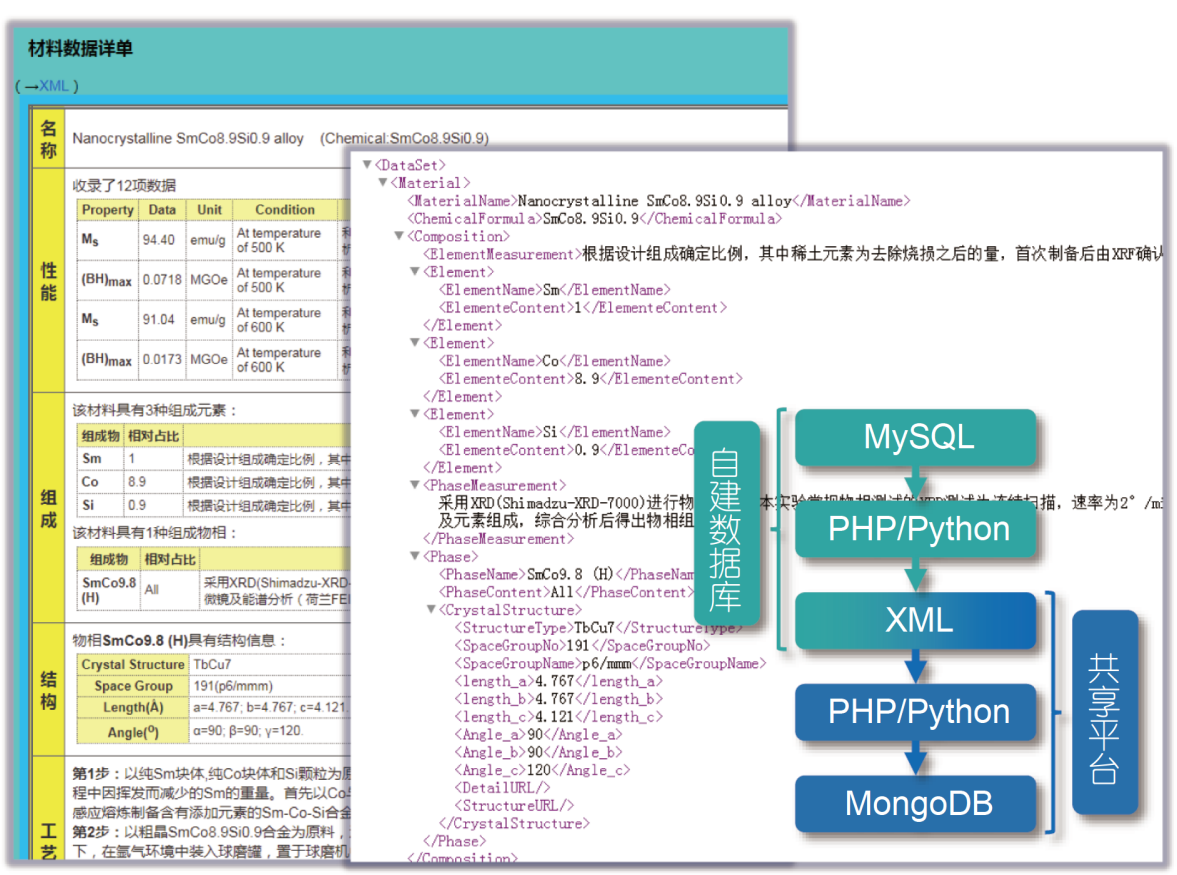

图 12 (网络版彩图)从系统中导出材料信息到XML文件进行数据汇交的示例

Figure 12 (Color online) An example of exporting material information from the system to XML file for data transfer.

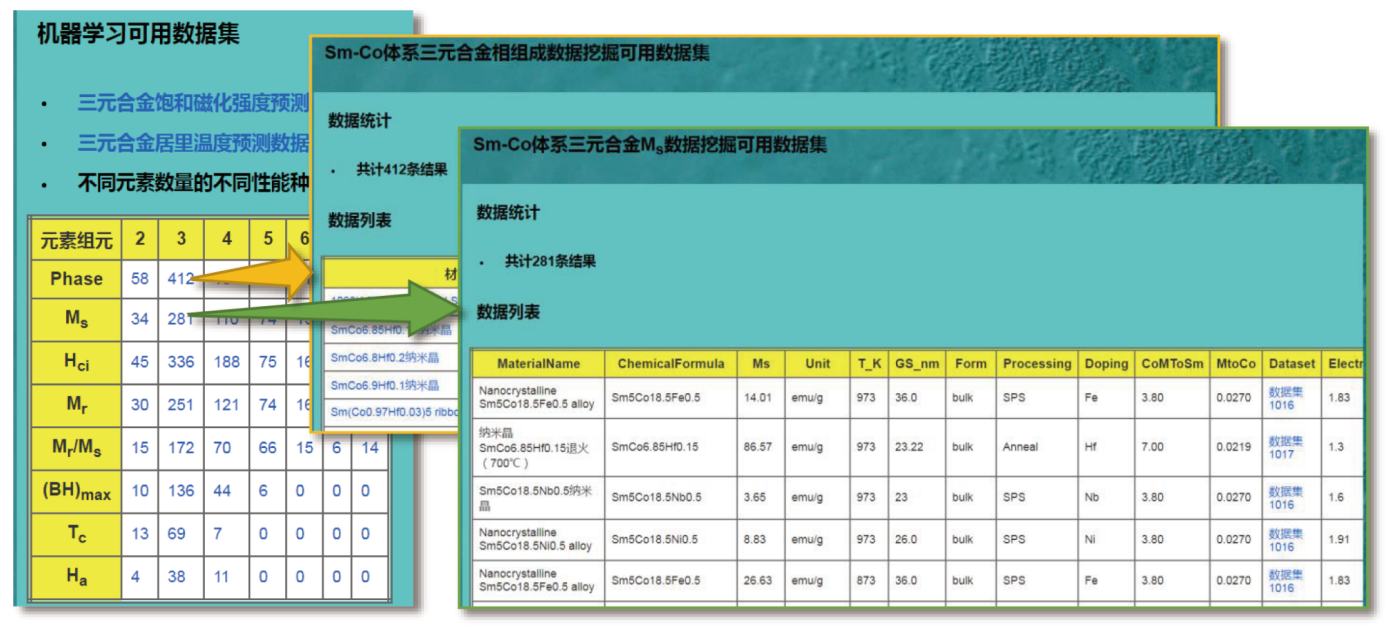

图 13 (网络版彩图)从系统中产生用于机器学习的数据集示例

Figure 13 (Color online) An example of generating datasets from the system for machine learning.

方法, 能够快速、充分地清洗数据, 获得的高质量数据 可应用到多个数据集中，这进一步加速了结合智能算 法开展的材料设计或知识发现进程.

基于专用数据库及信息管理系统，研究人员能够 在开展数据挖掘或机器学习时全面考虑各种变量的作 用，有效解决了构建数据集时经常面临的数据一致性 问题和数据误差问题. 理论上，若变量收集的足够充
分, 实验方案得到准确还原, 科学数据是可以被精确 复现的; 缺乏对关键变量的记录是多数数据集中出现 “数据不一致”现象的根源. 基于传统方法构建的数据 集在进行数据挖掘时, 若遇到变量缺乏的问题, 不得 不返回文献重新整理数据, 这一过程耗时耗力. 此外, 在数据误差方面, 以监督学习算法为例, 若自变量不够 丰富, 缺失的自变量对目标变量造成的影响不得不被 
研究者视为随机误差或系统误差，从而直接影响预测 精度. 而本研究建立的专用数据库首先对文献中出现 的各类数据项进行系统整理, 然后根据材料设计需求, 在数据分析软件中读取数据库, 按需调用并实时调整 变量, 从源头处理上避免上述问题. 由于本研究建立 的数据管理方案可以使研究人员同时阅览系统的材料 数据(即与该材料相关的各种因素), 从而可避免错过 对重要变量的分析, 并有效减小数据误差及其对目标 变量的影响, 由此加速数据挖掘的工作进程, 并使材 料设计的成功率得到提高.

综上，借助本研究建立的专用数据库及信息管理 系统，在构建材料设计专用数据集、开展数据挖掘时 具有如下优势: (1) 数据可在多个材料设计问题中重 复利用; (2) 对数据的清洗的结果可以被永久保留; (3) 可充分考虑多种因素对目标变量的影响; (4) 可根据数 据挖掘的进展情况快捷准确地调整变量.

\section{6 总结与展望}

面向数据驱动材料设计对高质量数据集的紧迫需 求, 我们针对研究文献这样极为重要的数据源, 构建了 满足材料基因工程应用的专用数据库及信息管理系 统. 针对多元合金通常具有的复杂制备工艺, 在有向无 环图数据结构的基础上设计模型，实现了复杂异构工 艺数据的融合，形成了可追溯的工艺数据存储形式. 针对多元合金体系通常具有多种性能指标和并存多种 单位制的情况，设计了规范的性能信息存储系统并在 其内实行单位制转换. 构建的数据关系模型集成了材 料元素组成、物相组成、晶体结构、制备工艺、性 能、文献等全面的数据，进一步开发了材料知识信息 解析、关联与管理系统，突破了传统数据库对海量数 据很难进行高效、准确的标注与管理等问题. 实践表 明，从数据源还原产生以往研究人员曾持有的小型数 据集的方法，对整合研究文献这种分散数据源中的材
料数据具有关键作用，不仅能够保证数据规范性，而 且显著提高数据标注的效率. 建立的面向数据驱动材 料设计的专用数据库, 可由机器或软件直接读取, 在 信息管理系统的辅助下，可提供材料设计研发过程中 涉及的多方面信息. 建立的专用数据库及信息管理系 统在材料深层次信息检索、与不同数据共享平台快速 汇交、满足材料设计的高质量数据集构建等方面获得 了实际应用.

就材料基因工程及数据驱动材料设计的未来发展 而言，专用数据库及其衍生的数据集、知识信息、材 料科学认知等必然在新的材料研发模式中发挥越来越 重要的作用. 以研究文献为主的数据源随着材料科学 与技术的迅速发展其储藏的材料数据愈加宝贵，以之 为源头建立各类材料体系的专用数据库及相应信息管 理系统必将更加重要, 其应用也将越来越广泛. 随着材 料研究文献逐年爆发式增长, 面向数据驱动材料设计 的专用数据库建设对结构化、规范化、批量化、多用 户协同等要求会越来越高，其数据体系与不同研究主 题下的数据集或数据共享平台的兼容汇交能力也应该 越来越强，以在更大的数据空间、以更高的效率持续 发挥作用. FAIR原则是材料基因工程专用数据库中对 收录和整理数据的最基本要求，在此基础上对专用数 据库中数据信息基于统一标准进行规范化治理，尤其 是针对研究文献这一庞大且极为重要的数据源关于数 据采集建立标准规范，是目前数据驱动材料设计发展 进程中非常值得重视的问题.

本文的数据库建设模式目前具有“高效采集、准 确标注、自动关联、同步协作”的特点, 实现了系统规 范化和收录自动化, 但仍离不开人工对文献的解读. 随 着标签化的数据达到一定规模, 可进一步借助强化学 习等智能算法对工作流程进行优化，从而使数据库内 容建设方式增加“智能提取、智能标注”等新的智能化 特点, 这将再一次加快对海量文献中材料数据的整理 工作, 并进一步提升材料数据的利用水平.

\section{参考文献}

1 Butler K T, Davies D W, Cartwright H, et al. Machine learning for molecular and materials science. Nature, 2018, 559: 547-555

2 Nosengo N, Ceder G. Can artificial intelligence create the next wonder material? Nature, 2016, 533: 22-25

3 Tropsha A, Mills K C, Hickey A J. Reproducibility, sharing and progress in nanomaterial databases. Nat Nanotech, 2017, 12: 1111-1114

4 Möller J J, Körner W, Krugel G, et al. Compositional optimization of hard-magnetic phases with machine-learning models. Acta Mater, 2018, 
153: 53-61

5 Ren F, Ward L, Williams T, et al. Accelerated discovery of metallic glasses through iteration of machine learning and high-throughput experiments. Sci Adv, 2018, 4: eaaq1566

6 Wen C, Zhang Y, Wang C, et al. Machine learning assisted design of high entropy alloys with desired property. Acta Mater, 2019, 170: 109-117

7 Xue D, Xue D, Yuan R, et al. An informatics approach to transformation temperatures of NiTi-based shape memory alloys. Acta Mater, 2017, 125: $532-541$

8 Lamport L. On interprocess communication. Distrib Comput, 1986, 1: 77-85

9 Rajan A C, Mishra A, Satsangi S, et al. Machine-learning-assisted accurate band gap predictions of functionalized MXene. Chem Mater, 2018, 30: 4031-4038

10 Lu S, Zhou Q, Ouyang Y, et al. Accelerated discovery of stable lead-free hybrid organic-inorganic perovskites via machine learning. Nat Commun, 2018, 9: 3405

11 Owolabi T O, Faiz M, Olatunji S O, et al. Computational intelligence method of determining the energy band gap of doped $\mathrm{ZnO}$ semiconductor. Mater Des, 2016, 101: 277-284

12 Seshadri R, Sparks T D. Perspective: Interactive material property databases through aggregation of literature data. APL Mater, 2016, 4: 053206

13 Kim E, Huang K, Saunders A, et al. Materials synthesis insights from scientific literature via text extraction and machine learning. Chem Mater, 2017, 29: 9436-9444

14 Kumar N, Rajagopalan P, Pankajakshan P, et al. Machine learning constrained with dimensional analysis and scaling laws: Simple, transferable, and interpretable models of materials from small datasets. Chem Mater, 2019, 31: 314-321

15 Zhang Y, Ling C. A strategy to apply machine learning to small datasets in materials science. npj Comput Mater, 2018, 4: 25

16 Buschow K H J. The crystal structures of the rare-earth compounds of the form $\mathrm{R}_{2} \mathrm{Ni}_{17}, \mathrm{R}_{2} \mathrm{Co}_{17}$ and $\mathrm{R}_{2} \mathrm{Fe}_{17}$. J Less Common Met, 1966, 11: 204208

17 Bakkalbasi N, Bauer K, Glover J, et al. Three options for citation tracking: Google Scholar, Scopus and Web of Science. Biomed Digit Libr, 2006, 3: 7

18 Rumble J R. Accessing materials data: Challenges and directions in the digital era. Integr Mater Manuf Innov, 2017, 6: 172-186

19 Gražulis S, Chateigner D, Downs R T, et al. Crystallography Open Database-An open-access collection of crystal structures. J Appl Crystlogr, 2009, 42: 726-729

20 Okamoto H, Massalski T B. Binary alloy phase diagrams requiring further studies. J Phas Equilib, 1994, 15: 500-521

21 Chun D M, Kim H J, Lee J C, et al. Web-based material database for material selection and its application programming interface (API) for CAD. Key Eng Mater, 2007, 345-346: 1593-1596

22 Kim M, Singh S P, Lee J W, et al. Identification of a narrow band red light-emitting phosphor using computational screening of ICSD: Its synthesis and optical characterization. J Alloys Compd, 2019, 774: 338-346

23 Tao F, Cheng J, Qi Q, et al. Digital twin-driven product design, manufacturing and service with big data. Int J Adv Manuf Technol, 2018, 94: 3563-3576

24 Dima A, Bhaskarla S, Becker C, et al. Informatics infrastructure for the materials genome initiative. JOM, 2016, 68: $2053-2064$

25 Wilson G, Aruliah D A, Brown C T, et al. Best practices for scientific computing. PLoS Biol, 2014, 12: e1001745

26 Wilkinson M D, Dumontier M, Aalbersberg I J J, et al. The FAIR guiding principles for scientific data management and stewardship. Sci Data, 2016, 3: 160018 


\title{
Development of database and information management system for data-driven materials design
}

\author{
SONG XiaoYan, LIU Dong, LIU XueMei, GUO Kai \& MAO Fei
}

Key Laboratory of Advanced Functional Materials, Ministry of Education of China, College of Materials Science and Engineering, Beijing University of Technology, Beijing 100124, China

With the progress of data-driven materials design, the scale and quality of datasets for intelligence algorithms such as machine learning and data mining have actually become the bottleneck for the practical benefit. The massive experimental data reported in the literatures and those unpublished are significant treasure trove for producing high-quality datasets. However, there has been a lack of specific database or information management system for storing and managing data as well as generating high-quality datasets. Using the Sm-Co alloy system as an example, which is a typical representative of the high-temperature permanent magnetic materials, we established an intelligent database and the corresponding information management system, where the data acquisition, data annotation, data extraction and data format conversion are flexibly integrated. We set up correlation models for the elemental composition, phase constitution, crystal structure, processing, measurement, and properties of the materials. From these models, the collected data are structured and the logical associations of related data are explicit. Thus, high-quality datasets can be produced from the developed system according to certain requirements for materials design. In the developed information management system, the online data annotation by multi-user has been realized. Moreover, the system is advanced in high-efficiency data entry and unifying data format, and precise search for redundant data. It has been confirmed that the present specific database and information management system play an important role in the data-driven materials design, and are promising in the applications in the fields of materials genome initiative and materials informatics.

materials database, information management system, materials genome initiative, data-driven materials design doi: 10.1360/SST-2019-0375 